\title{
MAXIMUM PRINCIPLE FOR A CERTAIN ELLIPTIC SYSTEM OF SECOND ORDER LINEAR EQUATIONS
}

The paper deals with a strong maximum principle for elliptic systems of the form (1). Certain sufficient conditions given in the paper are a generalization of the conditions for systems without mixed derivatives given in $[7]$.

\section{STRONG MAXTMUM PRINCIPLE}

Let $G$ denote an $n$-dimensional bounded domain in the euclidean space $E_{n}$, and $\dot{G}$ the boundary of the domain $G$. In the domain $G$ we consider the following system of equations:

$L[u] \equiv \sum_{i, j=1}^{n} a_{i j}(x) \frac{\partial^{2} u}{\partial x_{i} \partial x_{j}}+\sum_{i=1}^{n} B_{i}(x) \frac{\partial u}{\partial x_{i}}+c(x) u=F(x)$

where $u=\left(u_{s}\right)_{s=1}^{m}$ is a column vector, $x=\left(x_{1}, \ldots, x_{n}\right) \in G$, $a_{i j}(x)=a_{j i}(x)$ are continuous real functions, $B_{i}(x)$ and $C(x)$ are continuous real square matrices of degree $m$ and $\mathrm{F}(\mathrm{x})$ is a real column vector.

We assume that:

1) There exist numbers $\alpha_{0}>0$ and $\alpha_{1}>0$ such that for every $x \in G$ and for every system of real numbers $\lambda_{1}, \ldots, \lambda_{n}$ we have $\alpha_{0} \sum_{i=1}^{n} \lambda_{i}^{2} \leqslant \sum_{i, j=1}^{n} a_{i j}(x) \lambda_{i} \lambda_{j} \leqslant \alpha_{1} \sum_{i=1}^{n} \lambda_{i}^{2}$. 
2) $u(x)$ is a solution of system (1), regular in the closure $\bar{G}$ of the domain $G$, i.e. $u(x) \in C^{2}(G) \cap C^{\circ}(\bar{G})$.

Let $D(x)$ denote a real non-singular matrix of degree $\mathrm{m}$, regular in $\bar{q}$. Substituting to (1) $u=D v$, where $v=\left(v_{s}\right)_{s=1}^{m}$ is a column vector, we obtain:

$$
D^{-1} L[D v] \equiv \sum_{i, j=1}^{n} a_{i j} \frac{\partial^{2} v}{\partial x_{i} \partial x_{j}}+\sum_{i=1}^{n} B_{1}^{1} \frac{\partial v}{\partial x_{i}}+C_{1} v=D^{-1} F
$$

where $B_{i}^{1}=D^{-1}\left(2 \sum_{j=1}^{n} a_{i j} \frac{\partial D}{\partial x_{j}}+B_{i} D\right), C_{1}=D^{-1} I[D]$.

We introduce the following notation: $R(x)=\sqrt{(v, v)},(u, v)=$ $=\sum_{s=1}^{n} u_{s} \cdot v_{g}, e(x)$ - the unit vector (i.e. $\left.(e, e)=1\right)$ in the direction of the vector $v(x)$, defined for $R(x) \neq 0$.

We have $v(x)=R(x) e(x)$ and $R(x)=\sqrt{(v, v)}=\sqrt{\left(U^{-1} u, u\right)}$ where $U=D \cdot D^{T}$.

At every point $x \in G$, at which $R(x) \neq 0$, the function $R(x)$ satisfies the following equation ([1], [6])

$M[R] \equiv \sum_{i, j=1}^{n} a_{i j} \frac{\partial^{2} R}{\partial x_{i} \partial x_{j}}+\sum_{i=1}^{n}\left(B_{i}^{1} e, e\right) \frac{\partial R}{\partial x_{i}}+\Phi(x) R=\left(D^{-1} F, e\right)$

where $\Phi(x)=-\sum_{i, j=1}^{n} a_{i j}\left(\eta_{i}, \eta_{j}\right)+\sum_{i=1}^{n}\left(\left(B_{i}^{1}+2 b_{i}(x) I\right) \eta_{i}, \eta_{0}\right)+$ $+\left(c_{1} \eta_{0}, \eta_{0}\right), \eta_{0}=e, \eta_{i}=\frac{\partial e}{\partial x_{i}}$, I is the unity matrix of degree $m$, and $b_{i}(x)$ are arbitrary continuous functions in $G$.

From the inequality $\left|\left(D^{-1} \cdot \mathrm{F}, \mathrm{e}\right)\right| \leqslant \sqrt{\left(U^{-1} \mathrm{~F}, \mathrm{~F}\right)}$ we have

$$
M[R]+\sqrt{\left(U^{-1} F, F\right)} \geqslant 0 .
$$

Theorem (strong maximum princ i p I e)

I. If the form $\Phi(x) \leqslant 0$ in $G$, and $F \equiv 0$ then the function $R(x) \neq$ const. does not reach its positive maximum in the interior of $G$. 
II. If the form $\Phi(x) \leqslant-\mu_{1}<0$ in $G$ and the function $R(x)$ reaches a maximum at a point $x^{\circ}$ lying within $G$, then $R\left(x^{\circ}\right) \leqslant \sup _{x \in E} \frac{\sqrt{\left(U^{-1} F, F\right)}}{\mu_{1}}$.

III. If the form $\Phi(x) \leqslant 0$ in $G, F \equiv 0$ and the function $\mathrm{R}(\mathrm{x})$ f const. reaches 1ts positive least upper bound at a point $x^{1} \in \dot{G}$, the $\frac{\partial R}{\partial \nu}\left(x^{1}\right)<0$, for arbitrary nontangential inward direction $\nu$.

IV. If the form $\Phi(\mathrm{x}) \leqslant-\mu_{1}<0$ in $G$, the function $\mathrm{R}(\mathrm{x})$ satisfies the inequality $R(x) \geqslant \frac{\sqrt{\left(U^{-1} F, F\right)}}{\mu_{1}}$ in $G$ and reaches its positive least upper bound at a point $\mathrm{x}^{1} \in \dot{\mathrm{G}}$, then $\frac{\partial R}{\partial v}\left(x^{1}\right)<0$, for arbitrary nontangential inward direction $v$.

In theorems III and IV we assume additionally, that the point $x^{1}$ has the inside sphere property (i.e. there exists a closed sphere $K$ such that $K \subset \bar{G}$ and $K \cap \dot{G}=\left\{x^{1}\right\}$, see [2]) and the first derivatives of the solution of (1) and of the matrix $D$ are bounded in a certain neighbourhood of the point $x^{1}$.

Theorem I is formulated in [6], th. II in [7] and th.III in [3]. The proof of th. IV is analogous to that of th. III.

I e $\mathrm{m} m$ a. The form $\Phi(\mathrm{x}) \leqslant 0$ in $\mathrm{G} \Leftrightarrow$ there exists a real matrix $U(x)$, symmetric, positive definite and regular in $G$ and there exist functions $b_{i}(x) \quad(i=1, \ldots, n)$ continuous in $G$ such that for every $x \in G$ the matrix

$$
\begin{gathered}
I^{*}[U] \equiv 2 \sum_{i, j=1}^{n} a_{i j} \frac{\partial^{2} U}{\partial x_{i} \partial x_{j}}+4 \sum_{i=1}^{n}\left(B_{i}+b_{i} I\right) \frac{\partial U}{\partial x_{i}}+ \\
+\sum_{i, j=1}^{n} \bar{a}_{i j}(x)\left(B_{i}+2 b_{i} I\right) U\left(B_{j}^{T}+2 b_{j} I\right)+4 C U
\end{gathered}
$$

is nonpositive definite, $\bar{a}_{i j}(x)$ being the elements of the matrix inverse to $A(x)=\left(a_{i j}(x)\right)_{i, j=1}^{n}$.

P r o o f: We have

$$
\Phi(x)=-\left\|v^{-1} \eta-\frac{1}{2} v^{T} \widetilde{B}^{T} \eta_{0}\right\|^{2}+\frac{1}{4}\left(\tilde{c} \eta_{0}, \eta_{0}\right)
$$


where $\eta=\left(n_{i}\right)_{i=1}^{n}, \quad \widetilde{B}=\left(B_{1}^{1}+2 b_{1} I, \ldots, B_{n}^{1}+2 b_{n} I\right), \quad \tilde{A}=$ $=\left(a_{i j} I\right)_{i, j=1}^{n}, V$ is a solution of the matrix equation $V \cdot V^{T}=$ $=A, \widetilde{C}=2 C_{1}+2 C_{1}^{T}+\sum_{i, j=1}^{n} \bar{a}_{i j}\left(B_{i}^{1}+2 b_{i} I\right) \cdot\left(\left(B_{j}^{1}\right)^{T}+2 b_{j} I\right)$, $\|\eta\|=\sqrt{(\eta, \eta)}$.

Hence $\Phi(x) \leqslant 0$ iff the matrix $\tilde{c} \leqslant 0$. We have $D \tilde{C} D^{T}=$ $=\frac{1}{2}\left[\mathrm{~L}^{*}[\mathrm{U}]+\left(\dot{\mathrm{L}}^{*}[\mathrm{U}]\right)^{\mathrm{T}}\right]$. Hence the thesis of the lemma follows directly.

\section{INEQUALITYY IN THE MAX NORM}

We consider the boundary problem in a domain $G$ with the boundary $\dot{G}$ :

$$
\begin{aligned}
& \mathrm{L}[\mathrm{u}]=\mathrm{F} \quad \text { for } \mathrm{x} \in \mathrm{G} \\
& I_{1}[u] \equiv \frac{\partial u}{\partial v}+N(x) u=g_{1}(x) \quad \text { for } \quad x \in \Gamma_{1} \\
& u=g_{2}(x) \quad \text { for } x \in \Gamma_{2}
\end{aligned}
$$

where $\mathbb{N}(x)$ is a real square matrix of degree $m, \nu$ is a nontangential to $\Gamma_{1}$ inward direction, $\Gamma_{1}$ and $\Gamma_{2}$ are surfaces such that $\Gamma_{1} \cup \Gamma_{2}=\dot{G}, \Gamma_{1} \cap \Gamma_{2}=\varnothing, B_{1}(x)$ and $g_{2}(x)$ are continuous real vectors. We assume that every point $x \in \Gamma_{1}$ has the inside sphere property.

Let us assume that there exists a solution $u(x) \in C^{2}(G)$ $n C^{\circ}(\bar{G}) \cap C^{1}\left(G \cup \Gamma_{1}\right)$ of the problem $(1),(5),(6)$.

We substitute $u=D v$ into (5) and (6). Similarly as it has been done for the system (1) we multiply each member by the matrix $D^{-1}$ then substitute $\mathrm{v}=\mathrm{eR}$ and take the scalar product of the both sides with the vector $e$.

At every point $x \in \Gamma_{1}$, at which $R(x) \neq 0$, the function $R(x)$ satisfies the inequality 
$M_{1}[R] \equiv \frac{\partial R(x)}{\partial v}+\frac{1}{2}\left(D^{-1} I^{* *}[U]\left(D^{-1}\right)^{T} e, e\right) R(x) \geqslant-\sqrt{\left(U^{-1} g_{1}, g_{1}\right)}$,

where $I^{* *}[U] \equiv \frac{\partial U}{\partial \nu}+2 N U$. At every point $x \in \Gamma_{2}$ we have

$$
R(x) \leqslant \sqrt{\left(U^{-1} g_{2}, g_{2}\right)} \text {. }
$$

$\mathrm{T} h \mathrm{e}$ o $\mathrm{r}$ e $\mathrm{m}$. If there exist continuous functions $b_{i}(x)$, a symmetric matrix $U(x)$, positive definite and regular in $G$, and a positive constant $\mu_{1}$ such that

$$
I^{*}[U] \leqslant 0 \quad \text { for } \quad x \in G, \quad I^{* *}[U]+2 \mu_{1} U \leqslant 0 \text { for } x \in \Gamma_{1}
$$

then for the solution of the boundary problem $(1),(5)$, we get the estimates:

$$
\text { 1) } \sqrt{(u, u)} \leqslant K\left(\sup _{x \in G} \sqrt{(F, F)}+\sup _{x \in \Gamma_{1}} \sqrt{\left(g_{1}, g_{1}\right)}+\sup _{x \in \Gamma_{2}} \sqrt{\left(g_{2}, g_{2}\right)}\right)(9)
$$

the constant $K$ depending on $I, U, \mu_{1}, G$,

2) if moreover there is a positive constant $\mu$ such that $I^{*}[U]+4 \mu \mathrm{U} \leqslant 0$ for $\mathrm{x} \in \mathrm{G}$, then

$$
\begin{gathered}
\sqrt{\left(U^{-1} u, u\right)} \leqslant \max \left\{\sup _{x \in G} \frac{\sqrt{\left(U^{-1} F, F\right)}}{\mu}, \sup _{x \in \Gamma_{1}} \frac{\sqrt{\left(U^{-1} g_{1}, g_{1}\right)}}{\mu_{1}},\right. \\
\left.\sup _{x \in \Gamma_{2}} \sqrt{\left(U^{-1} g_{2}, g_{2}\right)}\right\} .
\end{gathered}
$$

The function $R(x)$ satisfies (4), (7), (8). The proofs of inequalities (9), (10) are similar to those given for a single equation in [4]. These inequalities for some special systems are also given in $[6-7]$.

\section{EXAMPLES OF THE MATRICES $U(x)$}

$E x$ a $m I e$ 1. We assume additionally: $C(x) \leqslant 0$, $B_{i}(x)=B_{i}^{T}(x)$, there exist positive constants $\beta_{i}$ such that 
$\alpha_{1} \beta_{i} \cdot I \leqslant B_{i}(x) \leqslant\left(\alpha_{1}+4 \alpha_{0}\right) \beta_{i} \cdot I$. Choose $U(x)=\exp \left(-\sum_{i=1}^{n} \beta_{i} \cdot x_{i}\right) \cdot I$ and $b_{i}=-\frac{1}{2} \beta_{i} \alpha_{1}$. Now we have

$U^{-1} I^{*}[U] \leqslant \sum_{i=1}^{n}\left[\frac{1}{\alpha_{0}} B_{i}^{2}-\left(4+\frac{2 \alpha_{1}}{\alpha_{0}}\right) \beta_{i} \cdot B_{i}+\left(4 \alpha_{1}+\frac{\alpha_{1}^{2}}{\alpha_{0}}\right) \beta_{i}^{2} \cdot I\right] \leqslant 0$.

$\mathrm{E} \mathrm{x}$ a $\mathrm{m} \mathrm{ple}$ 2. Let us suppose additionally: $\mathrm{C}(\mathrm{x}) \leqslant 0$, $B_{i}(x)=B_{i}^{T}(x), \quad\left|x_{i}\right|<r,-\frac{3 \alpha_{0}}{4 n r} \cdot I \leqslant B_{i}(x) \leqslant \frac{\alpha_{0}}{4 n r} \cdot I$. We select $U(x)=\left(n \cdot r+\sum_{i=1}^{n} x_{i}\right) \cdot I$ and $b_{i}=-\frac{3 \alpha_{0}}{8 n r} \cdot$ The above assumptions yield

$$
I^{*}[U] \leqslant \frac{2 n r}{\alpha_{0}} \sum_{i=1}^{n}\left[B_{i}^{2}+\frac{\alpha_{0}}{2 n r} \cdot B_{i}-\frac{3}{16}\left(\frac{\alpha_{0}}{n r}\right)^{2} \cdot I\right] \leqslant 0 .
$$

$\mathrm{E} \times \mathrm{am} \mathrm{m} I \mathrm{e}$ 3. Let us consider a system of two equations of the form $\Delta u+c u=0$, where $c=\left[\begin{array}{rr}0 & -1 \\ 0 & 0\end{array}\right]$. Let $b_{i}=0$ and $U=\left(u_{i j}\right)_{i, j=1}^{2}$ where $u_{12}=u_{21}=0, u_{11}=a_{2}+$ $-a_{3} x_{1}^{2}, \quad u_{22}=a_{1}-x_{1}^{2}$. From the condition $I^{*}[U] \leqslant 0$ it follows that the constants $a_{1}, a_{2}, a_{3}$ should be chosen in such a way that the following. inequalities hold.in $G$ :

$$
\left(a_{1}-x_{1}^{2}\right)>0, \quad\left(a_{2}-a_{3} x_{1}^{2}\right)>0, \quad\left(a_{1}-x_{1}^{2}\right)^{2}-4 a_{3}<0 .
$$

$\mathrm{E} \times \mathrm{am} \mathrm{ple} \mathrm{4.} \mathrm{Let} \mathrm{G}$ denote a two-dimensional bounded domain lying in the half-plane $x_{2}>0$. In this domain consider the following system of equations

$$
\frac{\partial^{2} u}{\partial x_{1}^{2}}+x_{2}^{s} \frac{\partial^{2} u}{\partial x_{1}^{2}}+B_{1} \frac{\partial u}{\partial x_{1}}+B_{2} \frac{\partial u}{\partial x_{2}}+c u=0
$$

where $0<s<1$. We assume that the boundary $\dot{G}$ of $G$ consists of a finite number of curves lying on the half-plane $x_{2}>0$ and of some line segments on the axis $0 x_{1}$. The domain $G$ is 
contained in the rectangle: $-r<x_{1}<r, 0<x_{2}<1$, where $r$ is a definite positive number.

We take $U(x)=\left(r+x_{1}\right) \cdot \exp \left(\frac{\alpha}{s-1} x_{2}^{1-s}\right) \cdot I, \quad b_{1}=-\frac{3}{8 r}, b_{2}=0$. Hence we obtain: if there exists a negative number $\alpha$ such that $(2 \alpha-\sqrt{2 \alpha(\alpha-5)}) \cdot I \leqslant B_{2}(x) \leqslant(2 \alpha+\sqrt{2 \alpha(\alpha-5)}) \cdot I$ and $-\frac{3}{4 r} \cdot I \leqslant B_{1}(x) \leqslant \frac{1}{4 r} I$, then $I^{*}[U] \leqslant 0$.

The following property holds: if the domain $G$ and the coefficients of the system (11) satisfy the conditions formulated in this example, and mereover, if the boundary $\dot{G}$ and the doefficients are sufficiently regular, then the Dirichlet problem for system (11) has a solution.

The proof of this property is similar to the proof for a single equation ([5]).

$E \mathrm{x}$ a $\mathrm{m} \mathrm{p} \mathrm{I.e} \mathrm{5.} \mathrm{Let} \mathrm{the} \mathrm{domain} G$ satisfy the coniitions from example 4. In this dcmain the following system of enuaticns is considered

$$
x_{2}^{s} \frac{\partial^{2} u}{\partial x_{1}^{2}}+\frac{\partial^{2} u}{\partial x_{2}^{2}}+B_{1} \frac{\partial u}{\partial x_{1}}+B_{2} \frac{\partial u}{\partial x_{2}}+C u=0,
$$

where $s \in(0,1) \cup(1,2)$.

Let $U(x)=\exp \left(\frac{\alpha}{s-2} \cdot x_{2}^{2-s}\right) \cdot I, \quad b_{1}=b_{2}=0$. Hence it follows that if there exists a real number $\alpha$ such that $\alpha>0$ for $0<s<1, \alpha<0$ for $1<s<2$ and the matrix

$$
\left[-2 \alpha(1-s) \cdot I+2 \alpha^{2} x_{2}^{2-S} \cdot I-4 \alpha x_{2} B_{2}+B_{1}^{2}+x_{2}^{S} B_{2}^{2}\right]
$$

is nonpositive definite in $\bar{G}$, then $I^{*}[U] \leqslant 0$.

Similarly as for the system (11), if the boundary $\dot{G}$ and the coefricients of (12) are sufficiently regular, then the boundary Dirichlet problem for the system (12) has a solution. 


\section{EST IMATION OF THE EIGENVALUES}

Consider the following eigenvalue problem:

$$
\begin{array}{ll}
I_{1}[u]+\lambda u=0 & \text { for } \\
I_{1}[u]=0 & \text { for } x \in \Gamma_{1} \\
u=0 & \text { for } x \in \Gamma_{2} .
\end{array}
$$

As beiore, we substitute $u=D v, v=e R$ into the systems (13), (14), (15) and multiply bcth sides by the matrix $\mathrm{D}^{-1}$. Then, we take the right-hand scalar product of both sides with vector $e(x)$, the scalar product being defined by $(u, v)=\sum_{i=1}^{n} u_{i} \cdot \bar{v}_{i}$, where $\vec{v}$ denotes the complex conjugate of $v$. At every point $x \in G \cup \Gamma_{1}$ at which $R(x) \neq 0$, the real function $R(x)$ satisfies the equations:

$$
\begin{aligned}
& \sum_{i, j=1}^{n} a_{i j} \frac{\partial^{2} R}{\partial x_{i} \partial x_{j}}+\sum_{i=1}^{n} \operatorname{Re}\left(B_{i}^{1} e, e\right) \cdot \frac{\partial R}{\partial x_{i}}+\operatorname{Re}(\Phi(x)+\lambda) R=0 \\
& \text { for } x \in G \\
& \frac{\partial R}{\partial \nu}+\operatorname{Re}\left(D^{-1} I^{* *}[U]\left(D^{-1}\right)^{T} e, e\right) \cdot R=0 \text { for } x \in \Gamma_{1} \text {. }
\end{aligned}
$$

Note that

$$
\begin{gathered}
\operatorname{Re} \Phi(\mathrm{x})=\Phi^{1}(\mathrm{x})+\Phi^{2}(\mathrm{x}) \\
\operatorname{Re}\left(D^{-1} \mathrm{~L}^{* *}[\mathrm{U}]\left(\mathrm{D}^{-1}\right)^{\mathrm{T}} \mathrm{e}, \mathrm{e}\right)=\sum_{\mathrm{S}=1}^{2}\left(D^{-1} \mathrm{~L}^{* *}[\mathrm{U}]\left(\mathrm{D}^{-1}\right)^{\mathrm{T}} \mathrm{e}_{\mathrm{S}}, \mathrm{e}_{\mathrm{S}}\right)
\end{gathered}
$$

where $e_{1}=\operatorname{Re}(e), \quad e_{2}=\operatorname{Im}(e)$, and $\Phi^{S}(x)$ denotes $\Phi(x)$ with substitution of $e_{g}$ for $e$.

$\mathrm{T} h \mathrm{e} \circ \mathrm{r} \mathrm{e} \mathrm{m}$ 2. Let $\mu$ be a real number. If there exists a positive definite symmetric matric $U(x)$ regular in $G$ and a positive constant $\mu_{1}$ such that

$$
I^{*}[U]+4 \mu U \leqslant 0 \text { for } x \in G, \quad I^{* *}[U]+2 \mu_{1} U \leqslant 0 \text { for } x \in \Gamma_{1}
$$


then $\operatorname{Re} \lambda>\mu$, where $\lambda$ is any eigenvalue of the problem (13), (14), (15).

P r o o f: By the Iemma we obtain

$$
\left[\Phi_{g}(x)+\mu \leqslant 0\right] \Longleftrightarrow\left[I^{*}[U]+4 \mu U \leqslant 0\right]
$$

Suppose that $(\operatorname{Re} \lambda-\mu) \leqslant 0$. From the above relation we have $\operatorname{Re}(\Phi(\mathrm{x})+\lambda) \leqslant 0$. Hence, making use of inequality (9) we obtain $u(x)=0$ a contradiction. Hence $\operatorname{Re} \lambda>\mu$.

For a single equation this theorem is given in [4]. If the coefficients of the system (1) satisfy the assumptions given in examples $1-5$ and $\dot{G}=\Gamma_{2}$ we have $\operatorname{Re} \lambda>0$.

5. MAXDMUM PRINCIPLE FOR A CERTAIN SYSTEM

OF INTEGRO-DIFFERENTIAL EQUATIONS OF ELLIPTIC TYPE

In a domain $G$ we consider the elliptic system ofintegro-differential equations

$$
L \cdot[u(x)]+\iint_{G} K(x, y) \cdot u(y) d y=0
$$

where $K(x, y)$ is given square matrix of degree $m$.

We assume there exists a solution of the system (16), regular in $\bar{G}$. At every point $x \in G$ at which $R(x) \neq 0$, the function $R(x)$ satisfies the equation:

$$
\operatorname{Mi}[R(x)]+\iint_{G}\left(D^{-1}(x) K(x, y) D(y) e(y), e(x)\right) \cdot R(y) d y=0 .
$$

Let us denote $K_{1}(x, y)=\frac{1}{4 \varepsilon} \cdot K(x, y) U(y) K^{T}(x, y)+\varepsilon U(x)$. We have $\mathrm{Mi}[R(x)]+\left(D^{-1}(x) \iint_{G} K_{1}(x, y) R(y) d y \cdot\left(D^{-1}(x)\right)^{T} e(x), e(x)\right) \geqslant 0(17)$ for any positive number $\varepsilon$. 
$\mathrm{T} h \mathrm{e} \circ \mathrm{r} \mathrm{e} \mathrm{m}$ 3. If there exist: a positive number $\varepsilon$, continuous functions $b_{1}(x)$ and a positive defintte symmetric matrix $U(x)$ regular in a bounded domain $\bar{G}$ such that

$$
I^{*}[U(x)]+4 \iint_{G} K_{1}(x, y) d y<0
$$

in $G$ then the function $R(x) \neq$ const. cannot reach its positive l.u.b. In the interior of $G$.

$P r \circ \circ f$. Suppose that the function $R(x)$ reaches its positive $1 . u . b$. at $a$ point $1 x^{\circ}$ of the interior of G. From the lemma and the supposition (18) we obtain

$\Phi\left(x^{0}\right)+\left(D^{-1}\left(x^{0}\right) \cdot \iint_{G} K_{1}\left(x^{0}, y\right) d y \cdot\left(D^{-1}\left(x^{0}\right)\right)^{T} \eta_{0}\left(x^{0}\right), \eta_{0}\left(x_{1}^{0}\right)\right)<0$.

We have

$\Phi\left(x^{0}\right) \cdot R\left(x^{0}\right)+\left(D^{-1}\left(x^{0}\right) \cdot \iint_{G} K_{1}\left(x^{0}, y\right) R(y) d y \cdot\left(D^{-1}\left(x^{0}\right)\right)^{T} \quad \eta_{0}\left(x^{0}\right), \eta_{0}\left(x^{0}\right)\right) \leqslant$ $\leqslant\left[\Phi\left(x^{0}\right)+\left(D^{-1}\left(x^{0}\right) \cdot \iint_{G} K_{1}\left(x^{0}, y\right) d y \cdot\left(D^{-1}\left(x^{0}\right)\right)^{T} \eta_{0}\left(x^{0}\right), \eta_{0}\left(x^{0}\right)\right)\right] R\left(x^{0}\right)<0$.

From this inequality, condition (17) and the maximum principle for elliptic operatora we obtain the contradiction. Hence theorem 3 holds.

In the case of a single equation of the type (16) the maximum principle is treated in [8].

REFERENCES

[1] А.В. Б и п а д ${ }^{l} 3$ е: 06 әлдиптических системах дифференциальных управненй с частными производными второго поpядка. ДокЛ. АНCCCP, (1957) 983-986.

[2] A. Fri e d m a $\mathrm{n}$ Partial Differeñtial Equations of Parabolic Type, Englewood Cliffs 1964. 
[3] Л.М. К у к с: Теоремы качественной теории систем второго порядка. Успехи матем. наук. 3 (1962) 181-184.

[4] H. H. Prot ter, H. F. Wein ber ger: Maximum Principles in Differential Equations, Englewood Cliffs 1967.

[5] М.М. С м и $р$ н о в: Вырождающие элдиптические и гипербодические уравнения. Москва 1966.

[6] T. s t y ś: Априорные оценки ретений одной линейной системы дифференциальных управлении... Bull. Acad. Polon. Sci.Ser. math.astron.et phys. (1965) 639-640.

[7] J. Wa s o w $\mathbf{k}$ 1: Maximum Principle for a Certain Strongly Elliptic System of Iinear Equation of Second Order. Bull. Acad. Polon. Sc1, Ser.math.astron.et phys. 12 (1970) 741-745.

[8] Л。М. Л и $\mathrm{x}$ т а $\mathrm{p}$ н и к о в: 0 принципе максимума некоторых кдассов управнений. Сибирск. матем. журн.8(1966) 465469.

Received February $1^{\text {st }}$, 1972.

Address of the Author: dr Janusz Wąsowski, Warszawa, ul. Sułkowskiego $6 \mathrm{~m} 2$. 
